\section{Emergence from anaesthesia: a winding way back}

Marco Cascella

Division of Anaesthesia and Pain Medicine, Istituto Nazionale Tumori, IRCCS Fondazione G. Pascale, Naples, Italy

Sir,

Accidental awareness with recall during general anaesthesia (AAWR) is a rare complication of general anaesthesia with an overall incidence of $\sim 1: 19,000$ anaesthetics [1]. About $20 \%$ of AAWR cases occur at the emergence from anaesthesia, while $90 \%$ of these events are potentially preventable, especially through the use of neuromuscular monitoring [1]. The remaining AAWR emergence episodes could be attributed to an error in anaesthesia management, such as inappropriate anaesthetic administration due to device malfunction or human error [2, 3]. In very rare cases, there is no readily identifiable cause. This letter chronicles the clinical presentation of an AAWR episode manifesting itself at the emergence from anaesthesia, in order to provide a picture of this rare complication (Table 1), and to underline how the pharmacodynamic and operating mechanism of general anaesthetics, and the relationships between consciousness and anaesthesia, pose a puzzle still too difficult to solve.

A 58-year-old Caucasian man $(86 \mathrm{~kg}, 178 \mathrm{~cm})$ affected by colon cancer was selected for elective laparoscopic left hemicolectomy. Before the beginning of the surgery, midazolam $2 \mathrm{mg}$ and fentanyl $100 \mu \mathrm{g}$ were administered as premedication. Anaesthesia was induced with intravenous propofol, titrated (total dose $140 \mathrm{mg}$ ) until loss of consciousness (LOC), along with fentanyl $100 \mu \mathrm{g}$. Anaesthesia maintenance was obtained through end-tidal concentrations (ETAG) of desflurane (in 40\% oxygen) with a minimum alveolar concentration (MAC) of 0.9. The ETAG-guide was achieved through a nomogram to estimate age, gender, weight, and height related MAC (Draeger Fabius apparatus).
Manually controlled remifentanil infusion was titrated on the patient's response. In addition, haemodynamic variations were managed through the guidance of the non-invasive cardiac output monitoring (NICOM ${ }^{\oplus}$ ) system. Neuromuscular blockade was obtained with cisatracurium $15 \mathrm{mg}$. The monitoring of the neuromuscular blockade was performed (NMT Trident Drager Infinity ${ }^{\circ}$ ) allowing the maintenance of the depth of anaesthesia (DoA) status (ETAG 0.9) until full reversal of the neuromuscular blockade. The operative course was uneventful and according to our standard anaesthetic approach, the ETAG value was maintained for the entire duration of the operation ( 2 hours and 15 minutes). At the end of surgery, remifentanil infusion was interrupted and extubation performed under recovery of the neuromuscular blockade (sugammadex $100 \mathrm{mg}$ ) with spontaneous breathing and the swallowing reflex. Moreover, we usually perform extubation under only a partial recovery of consciousness, usually corresponding to a MAC of about 0.2-0.3 with an anaesthetic expiration concentration ranging from 2 to $4 \%$, in the case of desflurane.

Although the recovery of consciousness at emergence was sudden, the patient immediately reported to the anaesthesiologist detailed conversations which had taken place between several professionals in the theatre. The report was so impressive as it described a conversation between two non-Italian visiting medical students speaking in English. The patient provided specific information about the duration of symptoms which lasted an estimated 3 to 5 minutes at the phase of awakening from anaesthesia.

The patient did not experience paralysis and the AAWR episode did not cause him concern and distress. Moreover, conversational psychodiagnostic interviews, at 1 and 6 months, demonstrated that the patient had no psychiatric sequelae.

The misuse of neuromuscular monitoring is a major risk factor for AAWR during emergence from anaesthesia [1], especially in case of butyrylcholinesterase deficiency [4]. However, a root cause analysis showed that the patient had

Table 1. Features of AAWR at the emergence from anaesthesia

\begin{tabular}{ll}
\hline Incidence & About 20\% of AAWR cases \\
Clinical Presentation & Paralysis and distress often reported \\
Causes & BChE deficiency (for succinylcholine and mivacurium) \\
& $\begin{array}{l}\text { Inadequate anaesthetic management (missed use of neuromuscular monitoring, awake extubation) } \\
\text { Equipment malfunction or human errors } \\
\text { Pharmaco-induction mechanisms (e.g., due to tobacco smoking, alcohol consumption, and centrally acting drugs) } \\
\text { Genetic resistance to anaesthetics }\end{array}$ \\
Avoidance strategies & $\begin{array}{l}\text { Common (depending on the distress, and AAWR duration) } \\
\text { Treatment }\end{array}$ \\
Maintenance of DoA until full reversal of neuromuscular blockade and avoid awake extubation, when possible
\end{tabular}

AAWR: accidental awareness with recall during general anaesthesia; BChE: butyrylcholinesterase; DoA: depth of anaesthesia 
no risk factors for AAWR, and had never undergone surgery. No equipment malfunction or human errors were detected, and neuromuscular monitoring was used. Moreover, a reversal agent (sugammadex) was administered.

On the other hand, in $16 \%$ of cases, the AAWR phenomenon has no easily recognizable cause [5]. Apart certain conditions, such as tobacco smoking, heavy alcohol consumption, and centrally acting drugs responsible for the so-called 'physiological resistance' to anaesthetic agents, probably through a pharmaco-induction mechanism, an innate or genetic resistance could be also possible. This is a fascinating and poorly understood pharmacodynamic phenomenon. For instance, preclinical experimental data, obtained from mutant analysis in Drosophila, demonstrated that a wide range of genes (e.g. encoding for secondmessengers, memory formation substrates, ion channels, synaptic proteins) and related isoforms are implicated in the normal response to anaesthetics [6].

However, the matter seems to be more complex. Indeed, emergence from anaesthesia is not simply the reverse process of induction as it is subjected to the control of different neural pathways [7] while, probably, several EEG patterns characterize the recovery of consciousness from DoA status [8].

Regarding the postoperative AAWR sequelae, a longer duration ( $>3-5$ minutes) of awakening has been associated with a higher incidence of psychological consequences, including posttraumatic stress disorder (PTSD) [1]. Probably, our patient did not present sequelae as he did neither manifested intraoperative distress nor discomfort in the immediate postoperative period. Furthermore, the psychological postoperative assessment helped to resolve any potential psychological consequence [2].

Could the complication be avoidable? Probably, the above-described AAWR episode was inevitable. Although the use of a DoA monitor (e.g., bispectral index, BIS) could have provided some help, compared with ETAG-guided anaesthesia, the use of BIS monitoring has not been associated with a reduced incidence of AAWR [9], while this complication has been reported even when BIS values [10] and ETAG concentrations were suggestive of a proper DoA status [9]. Moreover, the literature does not support routine BIS monitoring as part of standard practice [3] whereas, on the contrary, a recent large-sized clinical trial confirmed the results of previous studies [11] and showed that the use of ETAG-guided anaesthesia with a MAC ranging at 0.7-1.3 decreased the incidence of AAWR [12]. In conclusion, perhaps compared to the emergence phase, the induction and maintenance phases offer most opportunities for AAWR prevention, including the use of benzodiazepines (e.g., midazolam) as a premedication [13]. However, not all the AAWR events can be prevented, especially at emergence from anaesthesia which represents a very complex process with many dark sides still to be explained.

\section{ACKNOWLEDGEMENTS}

1. Source of funding: none.

2. Conflict of interest: none.

\section{References:}

1. Pandit JJ, Andrade J, Bogod DG, et al. Royal College of Anaesthetists, Association of Anaesthetists of Great Britain and Ireland, Royal College of Anaesthetists and the Association of Anaesthetists of Great Britain and Ireland. The 5th National Audit Project (NAP5) on accidental awareness during general anaesthesia: summary of main findings and risk factors. Anaesthesia. 2014;69(10): 1089-1101, doi: 10.1111/anae.12826, indexed in Pubmed: 25204236.

2. Cascella M, Viscardi D, Schiavone V, et al. A 7-year retrospective multisource analysis on the incidence of anesthesia awareness with recall in cancer patients: a chance of collaboration between anesthesiologists and psycho-oncologists for awareness detection. Medicine (Baltimore). 2016; 95(5): e2757, doi: 10.1097/MD.0000000000002757, indexed in Pubmed: 26844523.

3. American Society of Anesthesiologists Task Force on Intraoperative Awareness. Practice advisory for intraoperative awareness and brain function monitoring: a report by the american society of anesthesiologists task force on intraoperative awareness. Anesthesiology. 2006; 104(4): 847-864, indexed in Pubmed: 16571982.

4. Thomsen JL, Nielsen CV, Eskildsen KZ, et al. Awareness during emergence from anaesthesia: significance of neuromuscular monitoring in patients with butyrylcholinesterase deficiency. Br J Anaesth. 2015; 115 Suppl 1: i78-i88, doi: 10.1093/bja/aev096, indexed in Pubmed: 26174305.

5. Bergman IJ, Kluger MT, Short TG. Awareness during general anaesthesia: a review of 81 cases from the Anaesthetic Incident Monitoring Study. Anaesthesia. 2002; 57(6): 549-556, indexed in Pubmed: 12010269.

6. Al-Hasan YM, Krishnan HR, Ghezzi A, et al. Tolerance to anesthesia depends on synaptic proteins. Behav Genet. 2011; 41(5): 734-745, doi: 10.1007/s10519-011-9451-8, indexed in Pubmed: 21318409.

7. Kushikata T, Hirota K. Mechanisms of anesthetic emergence: evidence for active reanimation. Current Anesthesiology Reports. 2013; 4(1): 49-56, doi: 10.1007/s40140-013-0045-2.

8. Tarnal V, Vlisides PE, Mashour GA. The neurobiology of anesthetic emergence . J Neurosurg Anesthesiol. 2016; 28(3): 250-255, doi: 10.1097/ ANA.0000000000000212, indexed in Pubmed: 26274626.

9. Avidan MS, Zhang L, Burnside $B A$, et al. Anesthesia awareness and the bispectral index. N Engl J Med. 2008; 358(11): 1097-1108, doi: 10.1056/ NEJMoa0707361, indexed in Pubmed: 18337600.

10. Cascella $M$, Bifulco $F$, Viscardi $D$, et al. Limitation in monitoring depth of anesthesia: a case report. J Anesth. 2016; 30(2):345-348, doi: 10.1007/ s00540-015-2112-y, indexed in Pubmed: 26671259.

11. Avidan MS, Jacobsohn E, Glick D, et al. BAG-RECALL Research Group. Prevention of intraoperative awareness in a high-risk surgical population. N Engl J Med. 2011; 365(7): 591-600, doi: 10.1056/NEJMoa1100403, indexed in Pubmed: 21848460.

12. Wang J, Zhang L, Huang Q, et al. Monitoring the end-tidal concentration of sevoflurane for preventing awareness during anesthesia (MEETS-PANDA): A prospective clinical trial. Int J Surg. 2017; 41: 44-49, doi: 10.1016/j.ijsu.2017.03.015, indexed in Pubmed: 28323158.

13. Cascella M. Anesthesia awareness. Can midazolam attenuate or prevent memory consolidation on intraoperative awakening during general anesthesia without increasing the risk of postoperative delirium? Korean J Anesthesiol. 2015; 68(2): 200-202, doi: 10.4097/kjae.2015.68.2.200, indexed in Pubmed: 25844143.

\section{Corresponding author:}

Marco Cascella

Division of Anaesthesia and Pain Medicine, Istituto Nazionale Tumori, IRCCS Fondazione G. Pascale, Naples, Italy

e-mail:m.cascella@istitutotumori.na.it 